\title{
Welche Sicherheit und Rendite bietet die kapitalgedeckte Alterssicherung?
}

Zu Möglichkeiten der Risikoabschätzung

Der »Generationenvertrag « der gesetzlichen Rentenversicherung hat die Grenzen seiner Leistungsfähigkeit erreicht. Damit ist die »erste Säule« der Alterssicherung, die auf diesem Umlageverfahren basiert, ins Wanken geraten. Schuld daran ist die zunehmende Überalterung der Gesellschaft, aber auch die anhaltend hohe Arbeitslosigkeit, die zu enormen Beitragsausfällen führt. Schon heute sind die Rentenzahlungen nur noch zu rund 75 Prozent durch die Sozialversicherungsbeiträge der arbeitenden Bevölkerung gedeckt, der Rest muss - ähnlich wie bei den Beamtenpensionen - aus dem allgemeinen Steueraufkommen finanziert werden. Das birgt vor allem für die jungen Beitragszahler substanzielle Risiken. Angesichts dieser Perspektiven sind immer weniger junge Menschen bereit, steigende Rentenbeiträge bei stetig sinkenden Leistungen zu akzeptieren. Kann die kapitalgedeckte Alterssicherung diese Defizite auffangen? Wie lassen sich die vielfältigen Konzepte der privaten Alterssicherung bewerten?

Neben vielen Reparaturversuchen der gesetzlichen Alterssicherungssysteme spielen der Ausbau der »Zweiten« (betrieblichen) und »dritten« (privaten) Säule der Alterssicherung eine zentrale Rolle. Dabei handelt es sich um kapitalgedeckte Versorgungssysteme: Jedes Individuum baut während der Erwerbsphase einen eigenen Kapitalstock auf, aus dem später seine Ruhestandsleistungen finanziert werden. Mit einem zusätzlichen kapitalgedeckten Alterssicherungssystem sind verschiedene Vorteile verbunden: Es ist weniger anfällig gegenüber demografischen Veränderungen als die umlagefinanzierte gesetzliche Rentenversicherung, und das breite Produktspektrum bietet größere Flexibilität, da individuelle Aspekte wie Lebensalter, Risiko- und Zeitpräferenzen besser berücksichtigt werden können. Schließlich ist zu erwarten, dass eine Altersversorgung, die an die Wertentwicklung der internationalen Aktien-, Zinsund Immobilienmärkte gekoppelt ist, ein höheres Versorgungsniveau erreicht.

Diese Vorzüge wurden vom Gesetzgeber erkannt: Das im Mai 2001 verabschiedete Altersvermögensgesetz (AVmG) - auch unter dem Namen des damaligen Bundesarbeitsministers bekannt als »Riester-Rente « - fördert die kapitalgedeckte Alterssicherung, die das bisherige System ergänzt und teilweise auch ersetzt. Das Gesetz sieht vor, von Arbeitern, Angestellten sowie Beamten erbrachte Sparbeiträge für die Altersvorsorge steuerlich zu fördern und gleichzeitig das Leistungsniveau der gesetzlichen Rente beziehungsweise der Beamtenpension zu senken. Für Familien mit Kindern fällt die steuerliche Förderung besonders hoch aus. Im Gegensatz zur Versorgungsrücklage der Beamten fließen die Altersvorsorgegelder nicht in einen vom Staat (beziehungsweise von der Zentralbank) verwalteten »Kollektivfonds «, sondern in Altersvorsorgeprodukte, die von staatlich überwachten privaten Finanzinstitutionen wie Banken, 
Investmentgesellschaften und Versicherungen entwickelt und breiten Bevölkerungsschichten angeboten werden.

In einem solchen Marktumfeld steht der Verbraucher vor der Aufgabe, die verschiedenen Offerten zu beurteilen und aus dem reichhaltigen Produktspektrum eigenverantwortlich ein auf seine individuellen Bedürfnisse zugeschnittenes Altersversorgungsprogramm auszuwählen. Dabei darf eines nicht verschwiegen werden: Den Chancen der kapitalgedeckten Alterssicherung stehen auch Risiken gegenüber. So partizipieren die Anleger nicht nur an den Ertragschancen, sondern auch an den Verlustrisiken der Finanz- und Immobilienmärkte. Nach dem Fundamentalgesetz effizienter Finanzmärkte gehen höhere Renditechancen mit höheren Risiken einher. Für Anleger ist deshalb bei der Auswahl entscheidend, wie die langfristigen Rentabilitäts- und Risikopotenziale eines bestimmten Altersvorsorgeprodukts fundiert eingeschätzt werden können.

Welche wirtschaftswissenschaftlichen Methoden aus der betrieblichen Finanz- und Risikotheorie können dabei helfen? Der Beantwortung dieser Frage ist ein großer Teil der Forschung an der Frankfurter Investmentprofessur gewidmet. Einige der hierbei gewonnenen Erkenntnisse sollen im Rahmen der folgenden Fallstudie dargestellt werden.

\section{Langfristige Rendite- und Risikoprofile von Investmentsparplänen}

Kapitalanlage- und Versicherungsgesellschaften verwenden Investmentfonds als einen zentralen Baustein ihrer Altersvorsorgeprodukte. Diese Sondervermögen haben einen bestimmten Anlageschwerpunkt - etwa Aktien, Zinstitel, Immobilien. Erwirbt der Anleger solche Investmentanteile, partizipiert er daran, wie sich der Wert des Sondervermögens entwickelt.

Betrachten wir nun einen Altersvorsorgesparer, der zu Beginn eines jeden Jahres regelmäßige Sparraten in einen solchen Aktien-, Renten- oder Immobilienfonds beziehungsweise in eine Mischung dieser Basisfondstypen anlegt. Um den für die langfristige Altersvorsorge wichtigen Zinseszinseffekt möglichst uneingeschränkt ausnutzen zu können, sollen diese Fonds erwirtschaftete Erträge nicht ausschütten, sondern gleich wieder investieren (so genannte thesaurierende Fonds). Als Ausgabeaufschläge beim Erwerb der Fondsanteile werden pro geleisteter Sparrate 5 Prozent für den Aktien- und Immobilienfonds sowie 3 Prozent für Rentenfonds angesetzt, was in etwa marktüblichen Konditionen entspricht. Als Sparplandauer werden Horizonte von einem bis zu zwanzig Jahren betrachtet. Wie kann man nun ein Bild über die »Sicherheit « und die »Rendite« der betrachteten Fonds gewinnen?

In einem ersten Schritt ist zu klären, was unter Sicherheit beziehungsweise Risiko zu verstehen ist. Oft orientiert man sich dabei an der nominalen Kapitalerhaltung: Eine Anlageform ist umso sicherer, je geringer das Risiko ausfällt, am Ende weniger zu haben als eingezahlt wurde. Welches Risiko die Konzentration auf die nominale Kapitalerhaltung jedoch birgt, hat die Universität Frankfurt selbst in ihren Anfängen schmerzhaft erfahren müssen: Das Gründungskapital ging durch Inflation in den 1920er Jahren fast vollständig verloren. Nun soll hier keinesfalls das Gespenst einer 
Hyperinflation wie nach den beiden Weltkriegen an die Wand gemalt werden. Aber bei den für die Altersvorsorge relevanten langen Zeithorizonten sind auch die derzeit moderaten Inflationsraten beachtlich. Ein Beispiel: Bei einer Inflationsrate von 2 Prozent pro Jahr verliert ein unverzinslich angelegtes Anfangskapital nach 20 Jahren zirka 33 Prozent seines realen (Kaufkraft-)Werts. Da Sparen nichts anderes bedeutet, als Konsummöglichkeiten von der Gegenwart in die Zukunft zu verschieben, markiert der Ausgleich der Inflationsrate - und damit die reale Kapitalerhaltung - sicherlich die unterste Verteidigungslinie, an der sich die langfristige Rentabilität eines Altersversorgungsinstruments messen lassen muss. Aus diesem Grunde betrachten wir es in dieser Studie als Verlust, wenn die reale Kapitalerhaltung verfehlt wird.

\section{Wie lässt sich die Wahrscheinlichkeit eines Verlusts ermitteln?}

Im zweiten Schritt sind geeignete Kennzahlen festzulegen, mit denen das Risiko, einen realen Wertverlust zu erleiden, gemessen werden kann. Wir verwenden hierzu die beiden folgenden, auch intuitiv zugänglichen Kennziffern:

- Verlust-Wahrscheinlichkeit

- durchschnittliche Verlusthöhe im Verlustfall.

Die Verlust-Wahrscheinlichkeit gibt Antwort auf die Frage, bei wie vielen aller möglichen Wertentwicklungsszenarien die reale Kapitalerhaltung verfehlt wird. Sie gibt allerdings über das Ausmaß der möglichen Verluste keine Auskunft, denn eine Verfehlung um einen Euro wird genauso bewertet wie ein Verfehlen um 20.000 Euro. Diese Informationslücke schließt die zweite Risikokennzahl, welche die durchschnittliche Verlusthöhe erfasst - unter der Bedingung, dass die Inflationsrate als untere Verteidigungslinie verfehlt worden ist.

In einem letzten Schritt gilt es, ein Verfahren zu wählen, mit dem diese Risikokennzahlen quantifiziert werden können. Methodisch verwenden wir hierzu so genannte Monte-Carlo-Simulationstechniken. Dabei wird auf Basis eines geeigneten Modells für die Stochastik und die Dynamik der betrachteten Investmentfonds zufällig eine sehr große Anzahl alternativer Szenarien der zukünftigen inflationsbereinigten Wertentwicklungen erzeugt. Wir benutzen dafür die so genannte geometrische Brownsche Bewegung. Dieser Zufallsprozess geht auf den schottischen Botaniker Robert Brown (1773-1858) zurück, der damit die Bewegung von Pollen in einer Flüssigkeit beschrieb, und gehört heute zum Standardinstrumentarium in der Finanzmarktforschung und -praxis. Um eine möglichst hohe Präzision bei den Risikokennziffern sicherzustellen - insbesondere die Unterschätzung von Worst-Case-Risiken gilt es zu vermeiden -, werden jeweils 3.000.000 Simulationspfade betrachtet. Dies verlangt sehr leistungsfähige Rechner und eine »geschickte « Programmierungstechnik. Auf der Basis dieser 3.000.000 möglichen zukünftigen Wertentwicklungen werden nun die Verlust-Wahrscheinlichkeit - Anzahl der Wertentwicklungspfade, bei denen ein Verlust eintritt, geteilt durch die Anzahl aller Pfade (hier 3.000.000) - und der durchschnittlichen Verlusthöhe im Verlustfall berechnet.

In den Abbildungen [1], [2] und [3] sind die Simulationsergebnisse für Sparpläne in jeweils reinen Aktien-, Renten- und Immobilienfonds gegenübergestellt. Außerdem 
wird ein Mischfonds betrachtet, der sich aus 50 Prozent Aktien-, 35 Prozent Rentenund 15 Prozent Immobilienfonds zusammensetzt. Dies ist insofern wichtig, da die meisten Altersvorsorgeprodukte im Rahmen der »Riester-Rente« als Kombination dieser Hauptanlageklassen konzipiert sind. Bei bestimmten Investmentfonds (etwa bei Altersvorsorgesondervermögen) ist eine Streuung des Fondsvermögens über verschiedene Asset-Klassen hinweg sogar gesetzlich vorgeschrieben. Die Datenbasis zur Schätzung der Prozessparameter bezieht sich auf die realen Einjahresrenditen am Markt existierender Investmentfonds im Zeitraum 1980 bis 2001 mit durchschnittlicher Wertentwicklung.

Betrachten wir zunächst den zeitlichen Verlauf des mittleren Endvermögens der Investmentsparpläne [1]: Die Ertragsstärke der Aktienmärkte lässt (jedenfalls im Durchschnitt) das langfristig höchste Versorgungsniveau erwarten. Dabei steigt der mittlere Renditevorsprung des Aktienfonds relativ zum Immobilien-, Renten- beziehungsweise Mischfonds mit zunehmender Anlagedauer. So beträgt das mittlere inflationsbereinigte Endvermögen nach einer Spardauer von 20 Jahren beim Aktienfonds zirka 260 Prozent. In anderen Worten: beim Aktienfonds kann der Anleger nach zwanzig Jahren nahezu ein 2,6-fach höheres Konsumniveau erwarten als bei einer Anlageform, die eine Rendite exakt in Höhe der Inflationsrate aufweist. Bei einem reinen Immobilienfonds ergibt sich dagegen nur ein Wert von 145 Prozent. Sollte man also seine private Altersvorsorge somit ausschließlich auf Aktienfonds aufbauen? Lassen sich die demografischen Probleme der Alterssicherung durch die Ertragsstärke der Aktienmärkte so einfach beheben? Sind konservativere Anlageformen wie Immobilien- oder Rentenfonds für langfristige Anlagezeiträume überflüssig?

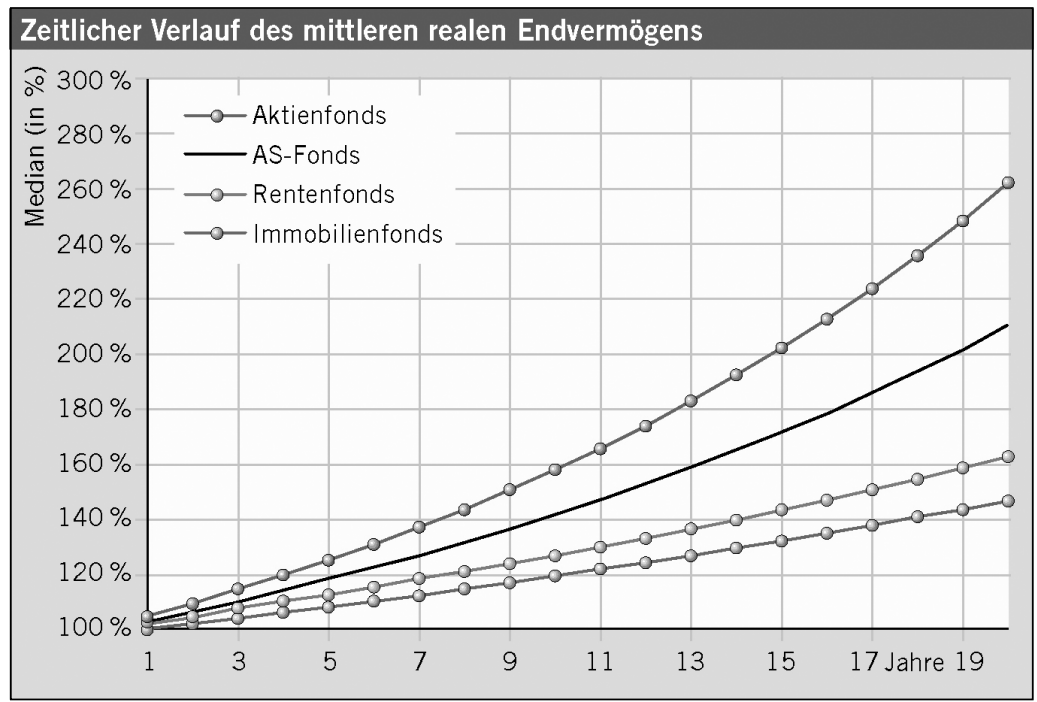

Bei der Interpretation der »mittleren Rendite « ist Vorsicht geboten: Denn die Hälfte der simulierten 3.000.000 zukünftigen Wertentwicklungsszenarien weist eine Rendite 
auf, die (unter Umständen deutlich) geringer als der Mittelwert ausfällt. Es stellt sich nun die Frage, in wie vielen Fällen sogar die Inflationsrate verfehlt wird, also sogar eine negative reale Rendite auf die Sparbeiträge erzielt wurde. Auf diese Frage gibt die Verlust-Wahrscheinlichkeit [2] Auskunft.

\section{Zeitlicher Verlauf der Verlustwahrscheinlichkeit (SW) relativ zur Referenzgröße} einer Kapitalerhaltung

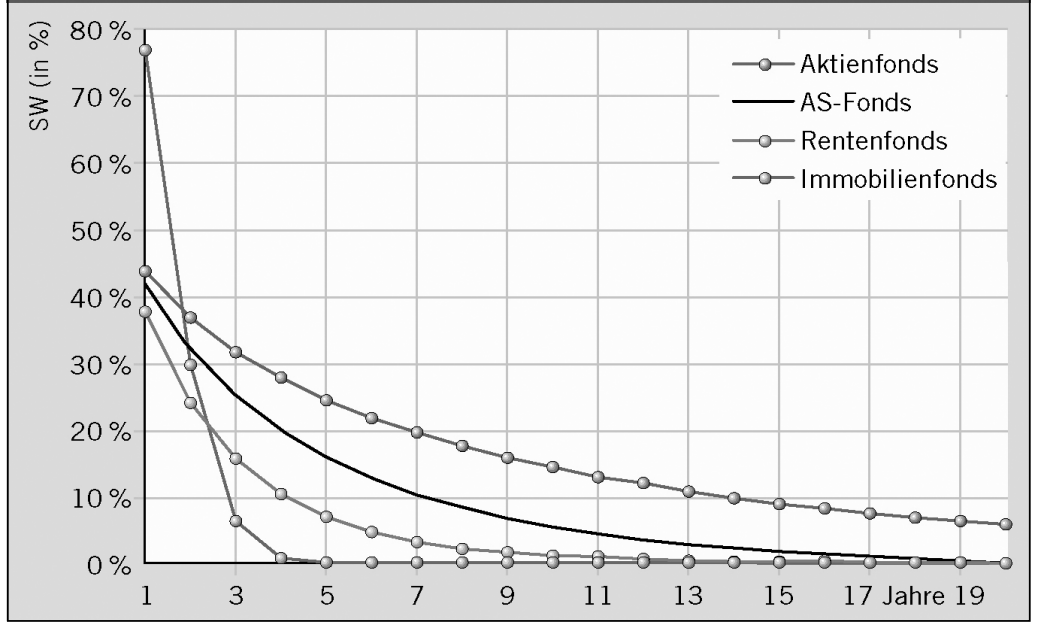

\section{Mischung macht's: Strategien gegen »Worst-Case»}

Abbildung [2] zeigt, dass - konsistent zum Fundamentalgesetz effizienter Finanzmärkte - Aktienfonds durchweg höhere Risikowerte aufweisen als die weniger ertragsstarken Immobilien-, Renten- und Mischfonds. Auffallend ist die sehr hohe VerlustWahrscheinlichkeit des Immobilienfonds bei einer Sparplandauer von nur einem Jahr, die dann sehr schnell (im dritten Jahr) auf geringe Werte abfällt. Dies ist maßgeblich auf den relativ hohen einmaligen Ausgabeaufschlag und nicht etwa auf Schwankungen des Fondsvermögens zurückzuführen. Auch bei den anderen Fondstypen nimmt das Risiko im Verlauf ab: bei Rentenfonds recht schnell, bei Aktienfonds eher langsam. Existiert also ein verlässlich wirkender zeitlicher Risikoausgleich-Effekt, bei dem mögliche Verluste eines bestimmten Jahres durch entsprechende Gewinne aus anderen Jahren kompensiert werden? Wenn dem so wäre, gibt es einen bestimmten Anlagehorizont, bei dem das Inflationsrisiko aus Aktienanlagen quasi vernachlässigbar gering ist?

Auch hier ist Vorsicht angebracht: Denn die Verlust-Wahrscheinlichkeit betrachtet nur die Anzahl der Fälle, in denen die Inflationsrate verfehlt worden ist. Wie hoch die Verluste damit im Einzelfall ausfallen, bleibt jedoch unbeantwortet. Auf diese Frage gibt der durchschnittliche Verlust im Verlustfall (DVV) [3] Antwort. Dabei werden nur diejenigen Fälle betrachtet, die in einem bestimmten Zeitpunkt die reale Kapitalerhaltung verfehlt haben. Für diese Verlustfälle wird dann der mittlere Unterschreitungs- 
betrag berechnet, insofern kann hier auch von einer Kennziffer für das Worst-CaseRisiko gesprochen werden.

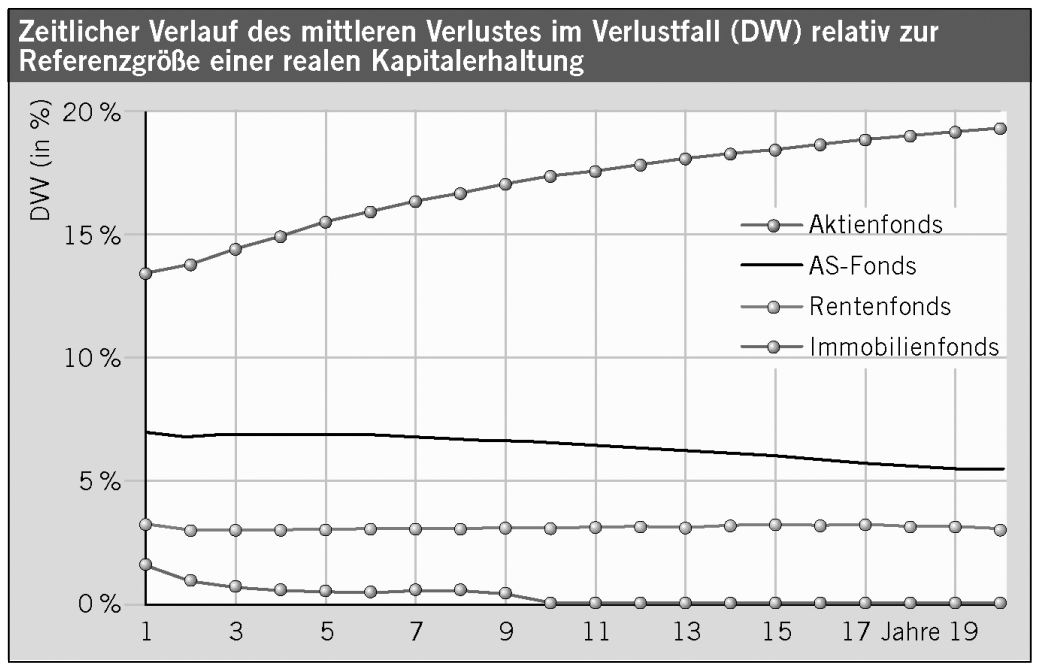

In der Worst-Case-Betrachtung sinkt mit zunehmender Sparplandauer das Risiko reiner Aktienfonds nicht. Vielmehr nimmt der durchschnittliche Unterschreitungsbetrag, und damit das Risiko, im Zeitablauf immer weiter zu. Damit wird aufgedeckt, wie gefährlich es ist, langfristig nur in Aktien zu investieren. Im Gegensatz dazu sinkt die Risikokennziffer beim Immobilien- und Mischfonds und bleibt beim Rentenfonds konstant. Insofern sind die im Vergleich zu Aktienfonds deutlich weniger ertragsstarken Immobilien-, Renten- und Mischfonds auch unter langfristigen Aspekten keine überflüssigen Anlageklassen. Vielmehr liegt in der hier gezeigten verlässlichen Kontrolle von Verlust-Risiken, insbesondere im Worst-Case-Bereich, die zentrale Stärke dieser Anlagen. In der Praxis machen sich die Anbieter von Altersvorsorgeprodukten die unterschiedlichen Chance-/Risikoprofile der betrachteten Fondstypen zunutze, da sie fast ausschließlich Mischfonds anbieten. Dabei kommt den Aktien die Rolle von »Renditetreibern « und Renten- und Immobilienfonds diejenige als »Risikostabilisatoren $\ll \mathrm{zu}$.

\section{Mehr Eigenverantwortung bedarf fundierter Kenntnis}

Für die kapitalgedeckte Alterssicherung gilt es, aus dem reichhaltigen, von Banken-, Investment- und Versicherungsgesellschaften angebotenen Produktspektrum eigenverantwortlich ein passendes Altersversorgungsprogramm zu entwickeln. Dabei gilt es, Chancen und Risiken abzuwägen, Abschlusskosten, Provisionen zu vergleichen, auf Produkttransparenz zu bestehen, nach Flexibilität und Dispositionsmöglichkeiten zu fragen und sich über die eigene Risikobereitschaft klar zu werden. All dies erfordert 
ein höheres Maß an Eigenverantwortung in Finanzfragen, das vielen noch fremd ist, aber trainiert werden kann. Vor dieser neuen Eigenverantwortung sollte man keine Angst haben. Ein Vergleich: Wer ein Auto fahren will, muss zunächst »Theoretisches « wie »Praktisches« lernen und üben. Beim Autokauf gilt es, komplexe Vergleiche von Preis-/Leistungsverhältnissen anzustellen, und wer schließlich die Vorzüge des Autofahrens genießt, muss Vorsicht und Verantwortlichkeit zeigen, um »Schadenrisiken « möglichst zu vermeiden. Trotz dieser Mühen und Risiken, wer ist hier zu Lande schon bereit auf das private Autofahren zu verzichten und nur öffentliche Transportmittel zu benutzen?

Aus einer wirtschaftswissenschaftlichen Perspektive sind mit der Chance-/Risikoabschätzung von Alterssicherungsprodukten viele spannende Fragen verbunden. In diesem Beitrag wurde der Frage nach den kurz-, mittel- und langfristigen Ertrags- und Risikoprofilen von Aktien-, Zins- und Immobilienfonds nachgegangen. Viele Fragen bleiben noch offen. Wie sind die Risiken und die Chancen der privaten Altersvorsorge in der Ruhestandsphase geeignet zu quantifizieren, wie lassen sie etwa Rentenhöhe zu Lebenszeiten und Vererbungsmöglichkeiten an die Nachkommen sinnvoll gegeneinander abwägen? Wie ist die Zufallsgesetzmäßigkeit der Wertentwicklung verschiedener Vorsorgeprodukte geeignet zu modellieren? Welche Rolle spielt das private Wohnungseigentum, und wie sieht ein effizientes Portfolio aus den verschieden Altersvorsorgeprodukten über den gesamten Lebenszyklus aus? Welche Effekte gehen von institutionellen Rahmenbedingungen wie Steuer- und Aufsichtsrecht aus? Es ist somit noch ein erheblicher Forschungsbedarf zu konstatieren.

\section{Anmerkungen}

Der Beitrag basiert auf früheren Arbeiten des Verfassers zu diesem Thema - unter anderem:

Raimond Maurer und Christian Schlag (2001): Erwartetes Versorgungsniveau und ShortfallRisiken von Investmentfonds-Ansparplänen, in: Der Langfristige Kredit 2001, 440-445.

Peter Albrecht und Raimond Maurer (2000): 100\% Aktien zur Altersvorsorge? - Über die Langfristrisiken einer Aktienanlage, in: AbsolventUm Universität Mannheim (ed.): 1. Mannheimer Alumni-Tag, Mannheim, 241-271. 